\title{
Entre culture générale et enjeux des métiers d'ingénieur : la diversité des enseignements en sciences humaines et sociales à l'École Centrale de Lyon
}

\author{
Nicolas Hourcade ${ }^{\mathrm{a}}$ \\ Professeur agrégé de sciences sociales, Ecole Centrale de Lyon, France
}

\begin{abstract}
Résumé. Ce texte analyse le développement d'enseignements en sciences humaines et sociales à l'École Centrale de Lyon, établissement formant des ingénieurs généralistes. Très limités au milieu des années 1990, les cours de SHS constituent aujourd'hui un aspect significatif de la formation. La première spécificité de cette offre pédagogique est de comprendre un nombre relativement important de cours obligatoires, complétés par des activités optionnelles. Sa seconde particularité est de traiter de thématiques générales à côté de contenus classiques liés aux enjeux des métiers d'ingénieur. Sa troisième caractéristique est de s'appuyer sur une implication de l'équipe SHS dans les activités professionnalisantes, tout en les séparant des enseignements disciplinaires. Enfin, l'inscription des SHS à l'ECL s'effectue plus par l'enseignement que par la recherche, chaque membre de l'équipe étant rattaché à un laboratoire extérieur différent.
\end{abstract}

Depuis une quinzaine d'années, l'École Centrale de Lyon (ECL) a développé une part significative d'enseignements en sciences humaines et sociales (SHS). Alors que l'offre pédagogique en SHS était le parent pauvre de la formation à l'ECL au milieu des années 1990, son importance est aujourd'hui une particularité sur laquelle l'institution communique volontiers. Ce texte décrit et analyse cette expérience afin d'alimenter les débats, auxquels ce colloque a utilement contribué, sur le rôle des SHS dans les formations scientifiques. Dans ce vaste ensemble, les contours des SHS s'avèrent flous. Il importe donc de préciser que cet article se centre sur les enseignements en psychologie, philosophie et sociologie assurés par les intervenants en SHS de l'ECL - tout en établissant des liens avec l'économie et la gestion ou les activités présentées par l'école comme professionnalisantes. Les exemples mobilisés sont plus particulièrement tirés des enseignements en sociologie assurés par l'auteur de cette contribution.

La première partie de ce texte analyse l'apparition et le développement du cursus en SHS à l'ECL, en mettant en évidence les facteurs favorables. La deuxième présente les spécificités de l'offre pédagogique en SHS à l'ECL, en insistant sur la diversité des formes et des objectifs. La troisième dresse un bilan de cette expérience, en s'interrogeant sur le positionnement institutionnel des SHS dans les formations scientifiques et en évoquant également les perspectives en termes d'activités de recherche, lesquelles n'ont eu jusqu'à présent qu'une influence limitée sur l'inscription des SHS à l'ECL.

\footnotetext{
a e-mail : nicolas.hourcade@ec-lyon.fr
}

This is an Open Access article distributed under the terms of the Creative Commons Attribution License 4.0, which permits unrestricted use, distribution, and reproduction in any medium, provided the original work is properly cited. 


\section{L'émergence d'un cursus en shs a l'École Centrale de Lyon}

L'ECL est une école d'ingénieurs généraliste à laquelle la plupart des élèves accèdent après une classe préparatoire scientifique. Elle accueille aujourd'hui environ 380 élèves par promotion. Au sein d'un système d'enseignement supérieur français clivé et hiérarchisé, elle se situe juste en-dessous des écoles d'ingénieurs historiques, basées en région parisienne. Cette place et sa nature généraliste font que l'ECL a pour objectif de former des ingénieurs ayant une vision globale de leur métier et capables d'en saisir les différents enjeux, scientifiques, techniques, économiques, éthiques, politiques, sociaux, humains...Ce positionnement s'est renforcé depuis une quinzaine d'années, ce qui a favorisé le développement des SHS.

\subsection{Une entrée par la recherche pour développer une offre jugée insuffisante}

C'est par la recherche que l'équipe d'enseignement en SHS commence à se constituer, dans la deuxième moitié des années 1990. En 1996, un laboratoire pluridisciplinaire, ICTT (Interaction Collaborative, Téléformation, Téléactivités) s'installe à l'ECL où l'un de ses responsables, un informaticien, est en poste. Associant des scientifiques sociaux de diverses disciplines (psychologie, sociologie, économie, sciences du langage, sciences de l'information et de la communication...) et des informaticiens, ce laboratoire étudie les technologies de l'information et de la communication ainsi que leurs usages, notamment professionnels. À la même période, en novembre 1996, un important colloque « Humanités et grandes écoles », organisé par la Conférence des Grandes Ecoles (CGE, 1997), se tient à Lyon avec le soutien local de l'Institut National des Sciences Appliquées (INSA) de Lyon et aussi de l'ECL. Également en novembre 1996, le Comité National d'Évaluation de l'Enseignement Supérieur remet un rapport sur l'ECL (CNE, 1996) qui s'inquiète « de l'insignifiance actuelle des enseignements de culture générale dans le cursus, quand on connaît l'importance qu'elle est amenée à prendre dans la formation des futurs ingénieurs » (p. 117). Ce document souligne que la culture générale et humaine «paraît être le parent pauvre de la formation des centraliens alors que ces élèves ne semblent pas toujours avoir une perception fine de leur environnement humain, social et économique. (...) L'École n'offre que peu d'enseignements de nature à élargir le champ intellectuel de ses futurs ingénieurs » (p. 41). Il est donc recommandé à l'ECL de " compléter et de développer les actions déjà entreprises dans le domaine de la formation générale et humaine » (p. 124) en s'appuyant notamment sur l'installation récente d'ICTT dans ses murs (p. 35).

Cette évaluation accélère les transformations des structures de l'ECL alors en cours. En 1998, un département spécifique, CLES (Communication, Langages, Entreprise, Sports) est créé pour rassembler toutes les disciplines autres que les sciences pour l'ingénieur (SPI). La même année, une psychologue membre d'ICTT est recrutée comme maître de conférences (MCF) à l'ECL. Elle est chargée de développer les enseignements en SHS en lien avec la recherche. À partir d'un bilan de l'existant dans les établissements français et étrangers, elle construit une offre pédagogique qui s'appuie, d'une part, sur les synergies avec les recherches menées à ICTT et, d'autre part, sur des activités professionnalisantes, en lien notamment avec le réseau des diplômés. Outre l'ouverture d'un cours optionnel de communication, qui rencontre vite un large succès auprès des élèves, l'encadrement des stages est renforcé et une activité obligatoire de rencontres d'ingénieurs par chaque élève est mise en place. Surtout, ce projet pédagogique s'articule autour du développement du « projet d'études » (PE), un travail collectif que les élèves mènent alors pendant les trois premiers semestres de leur scolarité, par groupes de 5 . Les sujets de PE recouvrent toutes les disciplines enseignées à l'ECL : si la plupart porte sur des thématiques de SPI, certains s'inscrivent dans les domaines de compétences du département CLES. En lien avec ces PE, sont créés des «TD SHS », dispensés à l'ensemble de la promotion par petits groupes de 15 élèves (3 PE), ce qui permet d'avoir recours à des pédagogies actives et nécessite l'intervention de plusieurs membres d'ICTT. Ces TD abordent des sujets divers : techniques d'expression apportant des conseils pratiques 
comme des contenus théoriques en psychologie de la communication, conduite de réunion, organisation collective de l'équipe, évaluation croisée des compétences, exposés de stage ouvrier, débats sur des sujets de société, etc. Ils ne donnent pas lieu à une évaluation directe puisque les élèves sont notés sur l'ensemble de leur PE, la qualité de leurs soutenances (préparées lors des TD SHS) étant un des critères de notation.

L'entrée des SHS à l'ECL s'est ainsi effectuée autour de deux principes : une activité de recherche liée aux problématiques de l'école, tant en enseignement, les scientifiques sociaux d'ICTT étant spécialistes des technologies de la communication et du travail notamment des cadres, qu'en recherche, le laboratoire associant SPI et SHS ; et une activité d'enseignement mêlant activités professionnalisantes et contenus scientifiques.

\subsection{L'élaboration d'un programme d'enseignements spécifiques en SHS}

Ce développement des SHS à l'ECL est renforcé avec l'arrivée, à la fin des années 1990, d'une nouvelle équipe à la tête de l'école. La direction désire alors restructurer les enseignements et positionner l'ECL comme formant des "managers » de haut-niveau. Considérant que les élèves ont besoin d'apports en SHS pour mieux saisir les enjeux de leur métier, elle souhaite intégrer, au-delà des TD déjà existants, des enseignements en SHS dans le cursus. Afin de mettre en place ce projet, deux Attachés Temporaires d'Enseignement et de Recherche (ATER), l'une en philosophie, devenue ensuite MCF, et l'autre en sociologie, qui a poursuivi comme professeur agrégé (PRAG), sont recrutés en 2001, avec comme mission d'aider la direction et la psychologue déjà en poste à approfondir le programme d'enseignements en SHS.

Un groupe de travail sur les SHS, composé de la direction, de l'équipe SHS, d'enseignants en SPI et de représentants des élèves, est constitué pour concevoir ce programme. Ce groupe s'est avéré, d'une part, contraint par la nécessité d'intégrer les SHS dans le projet pédagogique porté par la direction, de respecter un équilibre entre les diverses matières et de tenir compte de ressources limitées, tant dans la taille de l'équipe SHS que dans les moyens financiers pouvant être mis en œuvre, mais, d'autre part, très libre dans ses réflexions sur les contenus envisageables. Si la présence d'enseignants en SPI dans ce groupe de travail peut a priori étonner, elle avait essentiellement pour fonction d'apaiser les tensions liées à l'introduction de cours de SHS, qui impliquait de diminuer la part des enseignements en SPI. En fait, l'équipe SHS a bénéficié, et bénéficie encore, d'une grande autonomie dans la définition de ses contenus. De plus, l'implication de tous les acteurs de l'école dans ce projet a sans doute contribué à sa réussite.

Quoi qu'il en soit, ce groupe de travail a effectué un choix fort, en décidant d'intégrer une part significative d'enseignements obligatoires en SHS alors que, dans de nombreuses formations scientifiques, ces cours sont essentiellement optionnels. Quatre modules sont ainsi inscrits dans le tronc commun que suivent les élèves dans la première partie de leur cursus : un module principal de $30 \mathrm{~h}$ d'initiation aux SHS et trois enseignements de $12 \mathrm{~h}$ chacun, en communication, éthique et sociologie des organisations, auxquels s'ajoutent plusieurs modules optionnels sur des thématiques variées. Pour limiter les coûts, il est décidé de privilégier les cours magistraux, ce qui présente néanmoins l'inconvénient d'entraver le recours à des pédagogies actives.

Au même moment, une Unité d'Enseignement Professionnelle (UE PRO) est constituée afin de rassembler les activités professionnalisantes déjà existantes (dont le PE et les TD associés) et d'en développer de nouvelles. Dans cette UE PRO, les élèves sont les principaux acteurs, les enseignants se contentant de les encadrer.

\subsection{Une approche généraliste dans une école généraliste}

Parallèlement, le laboratoire ICTT disparaît en 2006, du fait d'un manque de moyens et, plus largement, de la difficulté à faire vivre un laboratoire pluridisciplinaire dans un univers scientifique cloisonné 
et une structure de taille modeste quand le CNRS privilégie les grands laboratoires. De plus, si les thématiques de recherche des enseignants en SHS sont en partie classiques dans ce type d'établissement (travail des cadres, philosophie des sciences notamment), les membres de l'équipe traitent aussi des sujets de recherche sans liens avec l'école et ont un profil généraliste, ce qui favorise deux principales évolutions dans l'offre pédagogique en SHS à l'ECL. D'une part, les contenus scientifiques ont été séparés des apports pratiques. Les TD liés aux projets ont ainsi été redéfinis en leur enlevant tout contenu disciplinaire, en les recentrant sur des techniques d'expression et d'organisation et en les rebaptisant en conséquence (TD Expression et Organisation et non plus TD SHS). Cette clarification a paru nécessaire car certains élèves et collègues en SPI avaient tendance à assimiler les SHS à des techniques d'expression. D'autre part, des contenus de culture générale, sans lien direct avec une formation d'ingénieurs, ont largement été intégrés au cursus, d'autant que les représentants des élèves dans le groupe de travail y étaient favorables. Ainsi, c'est sur proposition des élèves qu'un module optionnel de sociologie politique a été créé.

Par conséquent, l'implantation des SHS à l'ECL s'est faite dans une perspective largement inverse à celle amorcée par le laboratoire ICTT (même si l'évolution s'est faite dans la continuité), avec un ancrage dans l'institution plus par l'enseignement que par la recherche, des liens relativement limités (même s'ils existent) entre nos cours et nos travaux de recherche, un cloisonnement des enseignements disciplinaires et professionnalisants et des collaborations avec nos collègues en SPI limitées aux activités professionnalisantes.

\subsection{Une communication institutionnelle sur l'importance des SHS à l'ECL}

Ainsi, les SHS sont désormais bien installées à l'ECL. Dans sa dernière évaluation, l'AERES (2010) note que "l'ECL se positionne comme une école d'ingénieurs généraliste à forte composante scientifique. Dans un contexte marqué par le débat "expert-manager ", l'ECL a choisi de ne pas développer la formation au management au détriment de la qualité scientifique. (...) Le tronc commun (...) vise à donner aux étudiants de solides connaissances dans les disciplines des sciences dites exactes, mais aussi humaines et sociales. " En conclusion du rapport, la formation est considérée comme "bien équilibrée ». Dans sa communication externe (visible notamment sur le site http://www.ec-lyon.fr/), la direction souligne que l'école "forme des ingénieurs généralistes accomplis, ouverts et dotés de l'éthique indispensable pour inventer les mutations répondant aux enjeux sociétaux du XXI siècle. Pendant 3 ans, les élèves-ingénieurs bénéficient d'une formation scientifique et technique d'excellence, complétée par une culture significative en sciences humaines et sociales, une pratique du travail en projet, une maîtrise de 2, voire 3 langues vivantes et une ouverture concrète à l'internationalet au monde de l'entreprise. » De la même manière, dans les informations transmises par l'ECL à la Commission des Titres d'Ingénieurs (CTI, 2013), la direction de l'établissement a tenu à indiquer parmi ses particularités que "à la rigueur d'un socle de compétences scientifiques et techniques, (l'ECL) a associé dès 1998 un département dédié aux sciences humaines et sociales qui dote les Centraliens de Lyon d'un sens critique, et leur permet d'appréhender rapidement les fonctions managériales et/ou internationales qui seront les leurs».

Cette situation découle d'incitations extérieures des instances évaluatrices et de la volonté forte, à un moment donné, de la direction de développer les SHS, orientation qui a été acceptée ou renforcée par les directions suivantes. L'ECL a ainsi progressivement développé une stratégie claire : réaffirmer une solide base scientifique, tout en favorisant l'ouverture d'esprit des élèves. Cette optique offre aux sciences humaines et sociales une place dans le cursus centralien forcément mineure mais néanmoins significative. Le volume horaire de l'UE SHS, comme celle d'économie-gestion, est ainsi inférieur à celui des UE en SPI : $72 \mathrm{~h}$ officielles (64 h de créneaux de cours, $4 \mathrm{~h}$ d'examens et $4 \mathrm{~h}$ de travail en autonomie) contre $96 \mathrm{~h}$ pour les UE de SPI qui apportent chacune 6 crédits ECTS contre 5 pour l'UE SHS et autant pour celle d'économie-gestion. 
Ainsi, la part des SHS et le nombre d'enseignants statutaires sont aujourd'hui relativement importants pour une école d'ingénieurs. De plus, les intervenants en SHS (deux MCF et un PRAG) sont plus nombreux que ceux en économie et en gestion (deux MCF), ce qui n'est pas fréquent dans les formations scientifiques. Depuis septembre 2013, une nouvelle PRAG de sciences sociales intervient tant en SHS qu'en économie afin de renforcer les liens entre ces deux composantes, désormais réunies dans une même équipe d'enseignement. Cette particularité est liée aux options prises par la direction et à la forte intégration des enseignants en SHS dans l'UE PRO et dans les structures de l'école, ce qui a permis de nous faire connaître de nos collègues et de travailler directement avec eux. Notre implication dans la vie quotidienne de l'ECL ainsi que dans plusieurs conseils ou groupes de travail nous a également fourni des arguments et des moyens pour défendre l'idée que des enseignants statutaires en SHS connaissant bien l'école sont plus à même que des intervenants extérieurs de proposer des contenus adaptés et d'assurer un suivi des élèves.

\section{Des contenus, des objectifs et des formes pédagogiques diversifiés}

Actuellement, les enseignements en SHS sont concentrés sur les deux premières années du cursus, qui regroupent le tronc commun (réduit aux trois premiers semestres) et un semestre électif où les élèves approfondissent de manière libre plusieurs thématiques. Les SHS sont moins présentes dans la troisième année (voir III), qui propose aux élèves une spécialisation, à travers le choix d'options scientifiques et d'activités «métiers ». Dans les deux premières années, les enseignements en SHS sont de trois ordres : les cours obligatoires de l'UE SHS au troisième semestre et des activités électives ou professionnalisantes réparties sur les quatre semestres.

\subsection{Une découverte des SHS pour tous}

L'UE SHS est structurée autour des trois thématiques identifiées par le groupe de travail il y a une dizaine d'années. La première consiste à appréhender des grands enjeux de société, à travers un cours de $34 \mathrm{~h}$ de «sciences humaines », croisant les regards de la psychologie sociale, de la sociologie et de la philosophie autour de la thématique «individus et société ». Cet enseignement permet d'aborder des notions fondamentales : socialisation, normes, déviances, inégalités, identité... Il vise à montrer aux élèves l'intérêt de dépasser leurs représentations sociales pour construire une connaissance objective et maîtrisée du monde social. L'examen final consiste en l'analyse d'un texte, l'un des objectifs du module étant que les élèves s'exercent à décrypter des textes d'actualité (scientifiques ou médiatiques). L'objectif de la deuxième thématique est de permettre aux élèves de comprendre les organisations dans lesquelles les ingénieurs évoluent, via un cours de 16 h intitulé « organisations, travail, management », le Conseil d'Administration de l'ECL nous ayant, à une époque, incités à indiquer nettement toutes les parties du cursus où les élèves sont initiés au management. Si ce cours, qui mêle sociologie, psychologie et un peu d'économie, n'apprend évidemment pas aux élèves à manager, il compte leur apporter une aide indirecte à leurs futures actions professionnelles, en leur faisant prendre conscience des formes d'organisation et de management dominantes, des transformations du travail et des métiers d'ingénieur ou encore des opportunités ou des tensions auxquelles ils pourront être confrontés. Par exemple, la notion de risques psychosociaux y est analysée. Ce cours met en perspective les expériences que les élèves ont déjà eues en entreprise (particulièrement leur stage ouvrier de fin de première année) et les modules «métiers » qu'ils aborderont en dernière année. Il établit également des liens entre le fonctionnement de l'économie et des entreprises et les enjeux éthiques des métiers de l'ingénieur qui constituent la troisième thématique de l'UE SHS. Lors du cours d' « éthique » de $10 \mathrm{~h}$, les élèves sont en effet amenés à s'interroger sur les différents types d'éthique et sur la déontologie professionnelle des ingénieurs, à partir d'études de cas. Les examens des cours de sociologie des organisations et 
d'éthique consistent en des questions de cours visant à vérifier la compréhension générale de chaque enseignement.

Ainsi, les contenus de l'UE SHS se situent à deux niveaux : culture générale et enjeux des métiers de l'ingénieur. L'objectif n'est pas de présenter les SHS de manière académique. Il est d'initier les élèves aux regards des sciences sociales et de la philosophie afin de leur fournir des outils théoriques et méthodologiques, censés les aider à appréhender le monde dans lequel ils vivent et à forger leur esprit critique. Il s'agit plutôt de leur apporter des modes de questionnement que des réponses toutes faites, pour qu'ils puissent interpréter de manière argumentée et documentée les situations sociales auxquelles ils sont confrontés.

\subsection{Diverses activités électives}

À côté des cours obligatoires, plusieurs possibilités sont offertes aux élèves pour approfondir les SHS. Ces activités facultatives s'effectuent en groupes plus restreints et permettent de proposer des pédagogies actives voire de l'accompagnement individuel.

Les modules optionnels du deuxième semestre de la deuxième année constituent la principale opportunité. Les élèves en choisissent cinq (de $32 \mathrm{~h}$ chacun) parmi une cinquantaine. Dans chaque créneau, au moins un enseignement en SHS est proposé. Un élève peut donc n'en prendre aucun. À l'inverse, quelques-uns ne suivent que des modules en SHS. En tout cas, nos cours rencontrent une attente des élèves puisqu'ils sont généralement remplis. L'enseignement de « sociologie des comportements politiques » se poursuit, de même que celui de "philosophie des sciences et des techniques ». Le module « relations sociales en entreprise » reprend celui de communication initialement placé dans le tronc commun et l'associe à un cours de gestion des crises assuré par un journaliste. Un module «droit et travail » combine approches juridique (grâce à un intervenant extérieur en droit), psychologique et sociologique du travail, en particulier des cadres. Un cours sur « les enjeux sociaux du développement durable » croise des perspectives philosophiques, sociologiques et économiques. En 2013-2014, deux nouveaux enseignements ont été inaugurés : l'un associe les regards d'enseignants en SHS et en langues (les deux équipes cherchant à renforcer leurs collaborations) sur le thème «mondialisation et transculturalités »; l'autre traite « des déchets et des hommes » dans une perspective mêlant histoire, sociologie et économie.

L'autre voie privilégiée d'approfondissement des SHS est le « projet d'application » qui court désormais sur toute la deuxième année. Dans la plupart des cas, il consiste à traiter collectivement un sujet industriel formulé par une entreprise, mais une cinquantaine d'élèves peuvent choisir de s'initier à la recherche, y compris en SHS, seul ou avec un autre élève, à raison de $4 \mathrm{~h}$ par semaine. Chaque enseignant pouvant n'encadrer qu'entre un et deux projets, le nombre d'élèves travaillant en SHS est limité. Mais le format, long, et le tutorat des élèves permettent de les amener à construire une problématique, à mener des recherches bibliographiques, à lire des textes scientifiques, à expérimenter les méthodes d'enquête en SHS (entretiens, observations, questionnaires, analyse de documents...) et à produire un rapport scientifique. Là aussi, les thèmes oscillent entre culture générale et enjeux de l'ingénieur, comme l'illustrent des sujets récents en sociologie : les relations sociales sur les chantiers du BTP ; l'aménagement urbain du nouveau quartier lyonnais Confluence ; le traitement médiatique des mouvements sociaux suscités par la réforme du système des retraites; les femmes dans le jazz; l'extrême droite lyonnaise ; la prostitution ; les stéréotypes de genre à l'école primaire... La MCF en psychologie oriente les élèves vers ses sujets de prédilection, parfois en lien direct avec ses propres activités de recherche : le travail à distance, l'activité des cadres, les femmes ingénieurs ou l'entrepreneuriat. Quant à ceux en philosophie, ils traitent généralement des sciences et techniques.

En première année, quelques projets d'études ont une optique de SHS. Au sein d'un projet plus global liant la ville d'Écully (où est situé le campus) à l'ECL, trois PE ont étudié les effets sociaux de la traversée de la ville par l'autoroute A6, en traitant des données, notamment de l'INSEE, en 
réalisant des entretiens et en effectuant une enquête quantitative qu'ils ont eux-mêmes administrée. Plusieurs PE ont aussi mené des enquêtes quantitatives sur divers aspects de la vie de campus. Depuis deux ans, d'autres ont mis en place des « cafés éthique » à la demande du directeur. Le principe est d'inviter deux intervenants de haut niveau et de les faire débattre sur des enjeux éthiques des métiers de l'ingénieur : finance, risque nucléaire, armement, OGM, intelligence économique...Plus largement, l'équipe SHS soutient l'organisation de conférences sur des thématiques de société, en partenariat avec les enseignants responsables des conférences et avec le Bureau des Humanités formé par les élèves. Elle s'implique également dans des activités culturelles en lien avec des institutions lyonnaises (comme le Musée d'Art Contemporain) et collabore avec la bibliothèque pour proposer aux élèves diverses ouvertures culturelles.

\subsection{Les activités professionnalisantes}

Les enseignants en SHS participent également activement à plusieurs activités professionnalisantes au sein de l'UE PRO. Ils interviennent dans les TD d'expression et d'organisation et ceux de gestion de projet liés aux PE, le tutorat de PE (sur des sujets en SHS comme évoqué précédemment, mais aussi sur l'organisation d'événements ou l'entrepreneuriat), les visites d'entreprises (en encadrant des groupes d'étudiants), l'enquête découverte (qui consiste en une restitution de rencontres effectuées par les élèves avec deux ingénieurs) ou les stages (en animant des séances où les élèves présentent leur stage ouvrier). Si les enseignements professionnalisants et disciplinaires sont séparés, ils ne sont pas sans liens, ne serait-ce que parce que, dans les deux cas, il s'agit de favoriser un recul critique de la part des élèves. De plus, notre implication dans les activités de l'UE PRO nous fournit l'occasion d'établir des ponts avec les enseignements en SHS.

En particulier, l'un d'entre nous, en collaboration avec une enseignante en SPI, a repensé l'encadrement pédagogique des stages d'exécution en exigeant autant que faire se peut que le stage soit de nature ouvrière, en demandant aux élèves de rédiger un rapport analysant cette expérience puis de la présenter devant un enseignant et quelques camarades, et en leur fournissant un document de consignes pour le rapport qui s'apparente à un guide d'observation. Le cours de sociologie des organisations, qui est planifié dans le semestre suivant le stage ouvrier, peut dès lors s'appuyer sur ce guide et sur les expériences des élèves ainsi que sur les visites d'entreprises et l'enquête découverte.

\section{Bilans, questionnements et perspectives}

S'il nous satisfait largement, le développement des SHS à l'École Centrale de Lyon est confronté à des difficultés, de natures très diverses, auxquelles l'équipe est plus ou moins parvenue à apporter des réponses satisfaisantes. Comment intéresser un public hétérogène aux SHS ? Comment s'implanter en troisième année, chasse gardée des options scientifiques et des activités professionnalisantes ? Comment s'impliquer positivement dans l'établissement ? Comment concilier les activités pédagogiques et les projets de recherche des membres de l'équipe SHS ?

\subsection{Une offre diversifiée pour contenter un public hétérogène}

Le public centralien est extrêmement hétérogène dans ses connaissances préalables en SHS comme dans son intérêt pour ces disciplines. Schématiquement, trois types d'élèves peuvent être distingués. Un premier type ne vient pas en cours magistral et ne choisit aucune activité optionnelle en SHS, par réticence envers ces thématiques. Un deuxième type d'élèves est, au contraire, très intéressé par les SHS, manifestant une forte attente par rapport à ces cours, certains affirmant que ceux-ci sont leur principal centre d'intérêt pendant leurs deux premières années d'école. Quelques-uns d'entre eux prétendent même avoir choisi l'ECL pour la place qu'elle accorde aux SHS. Enfin, un troisième type 
peut se mobiliser selon le contenu ou la forme des enseignements en SHS : ces élèves demandent à être convaincus.

Dès lors, la première question qui se pose est de définir quel public viser dans nos enseignements obligatoires. Faut-il ne pas se soucier du nombre d'élèves présents en cours et ne s'adresser qu'aux plus motivés ? Ou convient-il de chercher à toucher un maximum d'élèves ? Même si des débats demeurent entre nous, nous privilégions la seconde option et tentons d'initier la plupart des élèves aux SHS. Cependant, l'UE SHS est essentiellement composée de cours magistraux, forme pédagogique peu prisée des élèves qui la trouvent ennuyeuse et qui, quelle que soit l'UE, s'en dispensent volontiers afin d'alléger leur emploi du temps puisque ce sont les seuls enseignements où la présence n'est pas contrôlée. Il nous a donc fallu réfléchir à des manières d'animer les séances et de favoriser l'interactivité. Nous avons également essayé d'attirer les élèves en amphi en ne mettant à leur disposition que des supports de cours lacunaires. Nous pensions les frustrer ou les mettre en difficulté et les inciter ainsi à venir en cours magistral. Autant dire que cette stratégie n'a pas été couronnée de succès, avec, par exemple, des résultats particulièrement mauvais en 2012-2013 : 1/3 des élèves a dû passer la seconde session de l'UE SHS et une partie de la promotion n'avait manifestement rien retenu de nos cours auxquels elle n'avait jamais assisté.

Nous avons donc apporté deux changements à cette UE en 2013-2014. D'abord, proposer des documents de cours détaillés, permettant aux élèves de comprendre l'enseignement même sans y assister, afin qu'ils retiennent les idées essentielles et qu'ils soient incités à venir aux cours suivants. Ces supports, encore perfectibles, sont particulièrement utiles pour la cinquantaine d'élèves étrangers. Profitant de l'arrivée d'une nouvelle PRAG dans notre équipe, nous avons surtout instauré quatre TD (au cours desquels la présence est contrôlée), consacrés à des analyses de textes ou de vidéos par groupes d'une cinquantaine d'élèves. La forte satisfaction des étudiants par rapport à ces TD, attestée par les résultats de l'évaluation des enseignements par les élèves, et l'amélioration des performances aux examens nous incitent à poursuivre dans cette voie.

En fait, face à l'hétérogénéité du public centralien, notre principale réponse est de proposer une offre diversifiée en SHS, tant dans les disciplines présentées que dans les thèmes abordés ou les formes pédagogiques. Par exemple, au-delà d'un noyau commun d'élèves motivés par les SHS, ceux qui assistent au cours de sciences humaines et ceux qui viennent aux amphis de sociologie des organisations ne sont pas exactement les mêmes. Ce constat a confirmé notre volonté de proposer tant des cours abordant des sujets liés aux métiers d'ingénieur que des modules de culture générale. À leur âge et à ce stade de leur parcours, certains élèves aspirent à des cours non utilitaires. La variété de nos manières d'enseigner est aussi un avantage : nous maximisons ainsi les chances qu'un élève soit intéressé au moins par une partie de l'UE SHS.

Cette diversité se retrouve dans nos activités optionnelles, pour lesquelles la principale question est de définir le niveau d'autonomie des élèves en projet. Étant donnée leur méconnaissance préalable des SHS, faut-il les encadrer très étroitement pour s'assurer qu'ils parviennent à des résultats ou les laisser un peu plus libres au risque qu'ils se fourvoient, ce qui peut s'avérer aussi frustrant que formateur ? Nos réponses sont variables, selon le sujet, l'enseignant et les élèves.

\subsection{En troisième année, pas d'option, mais des Masters}

En troisième année, la part des SHS est bien moindre que dans les deux premières. Les cours scientifiques sont limités aux SPI, avec une toute petite place pour l'économie et la gestion à travers des enseignements d'économétrie, de stratégie d'entreprise ou d'aide à la décision. Les SHS sont quasiment absentes, à quelques heures près assurées par la psychologue de l'équipe dans un module d'informatique, dans la continuité des travaux d'ICTT. Quant aux modules « métiers », ils sont essentiellement assurés par des PAST, des professeurs associés travaillant également en entreprise (avec lesquels l'équipe SHS collabore dans l'UE PRO et au sein du département CLES). Quelques 
interventions en SHS y ont lieu, en particulier un module de $16 \mathrm{~h}$ de philosophie au sein du « métier » " recherche et développement ». Par ailleurs, notre MCF en psychologie coordonne les projets d'entrepreneuriat menés par un petit groupe d'élèves. Nous suivons également des Travaux de Fin d'Études (TFE), en lien avec nos spécialités et, généralement, avec les activités « métiers » des élèves. En revanche, les velléités d'introduction de modules optionnels disciplinaires en SHS se sont heurtées à deux objections : les SHS prennent déjà suffisamment de place dans les deux premières années et les activités «métiers » occupent une part importante de la troisième année. En clair, les enseignants-chercheurs en SPI entendent préserver à la fois un volume horaire suffisant pour leurs cours de spécialisation et un nombre également suffisant d'élèves dans leurs options afin notamment d'attirer des thésards. Ayant déjà le sentiment d'avoir perdu des heures et des élèves lors des dernières réformes pédagogiques, ils ne sont guère enclins à laisser de la place en troisième année aux SHS, lesquelles sont implicitement renvoyées aux activités professionnalisantes des «métiers ».

Cette faible présence des SHS en troisième année signifie-t-elle que les SHS sont largement absentes quand les enseignements sont véritablement importants pour les élèves ? Cette question, soulevée lors du colloque, appelle trois réponses. D'abord, pour compenser la difficulté à s'inscrire dans la troisième année, l'équipe SHS s'est attachée à développer des Masters. Trois sont actuellement proposés en complément de la scolarité centralienne. Un en psychologie sociale sur le travail coopératif et le travail en réseau (co-habilité avec Lyon-II), un en histoire, philosophie et didactique des sciences (conventionné avec Lyon-I) et un en éthique et développement durable (conventionné avec Lyon-III). Chaque année, une bonne dizaine d'élèves s'inscrivent dans l'un de ces Masters, ce qui permet aux plus motivés de poursuivre leurs études dans nos disciplines.

Ensuite, les deux premières années sont loin d'être moins importantes que la troisième - où l'entrée sur le marché du travail se fait plus pressante et où une part significative de la promotion n'est plus sur le campus (mais dans un autre établissement, en particulier à l'étranger). Ces deux premières années constituent un sas entre la classe préparatoire et la vie active, au cours duquel certains élèves ont envie de s'ouvrir à de nouveaux regards et sont réceptifs aux SHS. Cette ouverture aux SHS peut ensuite être prolongée, pour les plus intéressés, par un Master mené en parallèle de la troisième année ou par un TFE en lien avec nos disciplines, mais aussi par des formations complémentaires réalisées après leur sortie de l'école. Ainsi, tous les ans, plusieurs élèves nous demandent une recommandation pour suivre un Master en SHS.

Enfin, notre objectif principal en tant qu'enseignants en SHS dans une école d'ingénieur généraliste n'est pas de former des scientifiques sociaux ou des philosophes. Il est plutôt d'armer intellectuellement de futurs ingénieurs, à travers un socle culturel en SHS, et de les aider à prendre du recul par rapport à leur métier et au monde dans lequel ils vivent. Certes, quelques élèves deviennent philosophes des sciences, économistes ou chargés d'enquêtes sociologiques : nous leur offrons cette opportunité et les aidons dans ce changement d'orientation. Mais ces parcours sont marginaux. Notre attention doit être avant tout centrée sur la masse des élèves qui ne poursuivront pas dans nos disciplines, mais qui pourront être durablement marqués par nos cours.

Une telle perspective est vraisemblablement liée au positionnement généraliste de l'école et aux profils des enseignants en SHS, qui sont inscrits dans des laboratoires extérieurs à l'ECL et ne cherchent pas particulièrement à recruter des thésards (même si le Master de psychologie peut aussi remplir cette fonction). Des chercheurs en SHS intervenant dans une formation scientifique spécialisée, ayant des objets d'études liés aux problématiques de leur établissement et/ou souhaitant collaborer avec des laboratoires de SPI risquent d'avoir des centres d'intérêt, des objectifs pédagogiques et un positionnement différents des nôtres. D'ailleurs, nos interventions dans une formation en alternance d'ingénieurs en énergie récemment ouverte par l'ECL nous ont montré que les contenus et les formes pédagogiques construits pour les élèves ingénieurs généralistes ne s'appliquent pas tous à ce nouveau public, lequel nécessite d'imaginer d'autres approches. Naturellement, l'offre pédagogique en SHS dans 
les formations scientifiques est fortement liée au contexte particulier de l'école ou de l'université et aux profils des étudiants.

\subsection{Quelle inscription dans l'école?}

Même si certains collègues en SPI continuent d'assimiler les SHS aux techniques d'expression ou aux activités professionnalisantes ou estiment que les SHS prennent trop de place dans le cursus ou considèrent qu'il vaudrait mieux employer des vacataires au lieu de donner des postes de statutaires aux SHS, les SHS semblent plutôt bien acceptées dans l'institution. D'ailleurs, les enseignants en SHS sont associés aux formations récemment ouvertes par l'ECL : l'équipe intervient de manière significative dans la formation en alternance d'ingénieur en énergie et une anthropologue a été recrutée dans le Master IDEA (Innovation, Design, Entrepreneuriat, Arts) porté par l'ECL avec l'École de Management de Lyon voisine.

Outre notre investissement dans l'UE PRO et les structures de l'ECL, cette bonne intégration des SHS est peut-être aussi due à notre attitude. À mon arrivée à l'ECL, j'ai été frappé par l'hostilité de l'un des intervenants en SHS à l'égard de la direction et des collègues de SPI. Il réagissait dès que ceux-ci opposaient les sciences (en parlant des seules SPI) aux autres matières, ce qu'il interprétait comme une dénégation du caractère scientifique des SHS, il employait un vocabulaire jargonnant, il estimait que les SHS n'étaient pas suffisamment reconnues dans l'ECL, etc. Ce comportement m'a paru largement injustifié : au contraire, je trouvais l'ECL plutôt ouverte aux SHS. Surtout, il était contre-productif, en tendant les relations. L'équipe SHS de l'ECL s'est donc efforcée, au contraire, de s'inscrire positivement dans l'établissement, de participer aux projets collectifs, ce qui nous a parfois permis de les faire évoluer, et de considérer nos collègues en SPI comme des partenaires avec lesquels nous construisons la formation des élèves plutôt que comme des adversaires qui nous seraient forcément hostiles. Évidemment, cette vision positive est liée à un contexte particulier et favorable, puisque l'ECL est, du fait de son positionnement généraliste et de sa place dans la hiérarchie des écoles, disposée à accorder une place aux SHS et que les récentes directions de l'ECL étaient convaincues de l'intérêt des SHS et à l'écoute de nos attentes. Néanmoins, ce changement d'attitude de notre part a produit des effets positifs qui méritent d'être soulignés.

\subsection{Quelles perspectives pour la recherche?}

L'inscription des SHS à l'ECL s'est donc effectuée plutôt par l'enseignement. Néanmoins, même si l'équipe comprend désormais deux PRAG, l'activité de recherche des enseignants en SHS est valorisée. Elle est perçue comme une source de crédibilité scientifique dans un établissement où la recherche est fortement mise en avant. Elle permet aussi de former quelques élèves à la recherche en SHS, via les projets d'application puis les Masters. Par conséquent, l'équipe a opté pour un rattachement individuel de chacun de ses membres dans des laboratoires extérieurs à l'ECL et reconnus par le CNRS.

Récemment, tant la direction de l'ECL que l'équipe SHS ont réfléchi à des moyens de parvenir à mieux valoriser les activités de recherche en SHS en interne et à les mettre en cohérence avec l'école et ses laboratoires. Sans grandes conséquences pour l'instant et sans remise en cause de notre positionnement. En effet, il paraît difficile tant de constituer un laboratoire spécifique aux SHS à l'ECL, du fait de notre nombre limité, que de rattacher l'ensemble de l'équipe à un laboratoire extérieur, du fait de notre diversité.

Dans un milieu universitaire où la conciliation des activités d'enseignement et de recherche est loin d'être évidente, il est encore plus délicat pour nous de mettre en cohérence enseignement et recherche. Pour l'instant, chacun des enseignants en SHS s'accommode bien de cette situation, qui nous permet de poursuivre nos recherches sur nos sujets de prédilection dans un environnement disciplinaire, mais elle demeure une source potentielle de tensions. De même, du fait de la taille limitée de l'équipe, les 
perspectives de carrière en interne sont nécessairement réduites, d'autant que le contexte général de l'enseignement supérieur est peu favorable aux créations de postes ce qui renforce la concurrence en la matière avec les autres disciplines au sein de l'école.

L'expérience pédagogique en SHS à l'ECL présente donc de nombreux aspects positifs. Elle s'inscrit pleinement dans le tronc commun des élèves ingénieurs et fournit des possibilités significatives d'approfondissement aux plus motivés. Elle s'appuie sur une offre diversifiée, tant dans les contenus, mêlant culture générale et analyse des enjeux concrets des métiers d'ingénieur, que dans les formes pédagogiques, allant du cours magistral à l'accompagnement individualisé. Elle est cependant confrontée à plusieurs difficultés. Il est notamment difficile de toucher tous les élèves quand certains sont réfractaires aux SHS et aux cours magistraux. Paradoxalement, l'équipe SHS est aussi victime de son succès. La direction nous demande régulièrement de proposer de nouveaux projets ou d'assurer de nouveaux cours, ce qui tend le planning des enseignants de l'équipe et va sans doute nous obliger à faire des choix dans nos interventions.

\section{Sources}

- AERES (Agence d'Evaluation de la Recherche et de l'Enseignement Supérieur), 2010, Rapport d'évaluation de l'Ecole Centrale de Lyon, http://www.aeres-evaluation.fr/Etablis sements/ECOLE-CENTRALE-DE-LYON

- CGE (Conférence des Grandes Ecoles), 1997, Humanités et grandes écoles, actes du colloque de Lyon de novembre 1996.

- CNE (Comité National d'Evaluation de l'Enseignement Supérieur), 1996, Rapport d'évaluation de l'Ecole Centrale de Lyon, https: //www . cne-evaluation.fr/WCNE_pdf/ECLYON.pdf.

- CTI (Commission des Titres d'Ingénieur), 2013, Données certifiées de l'Ecole Centrale de Lyon, http://extranet.cti-commission.fr/recherche/showDonneesCertifiees/id/323

\section{Remerciements}

Cette expérience pédagogique et, par conséquent, cet article reposent sur l'investissement des membres de l'équipe SHS de l'ECL. Je tiens à remercier Jacqueline Vacherand-Revel, qui a initié cette aventure, Sarah Carvallo et Laure Flandrin, ainsi qu'Ariane Pierrou et tous ceux qui ont occupé des fonctions d'ATER, de moniteur ou de vacataire dans notre équipe. L'implication des directeurs et directeurs des études a également été décisive. Mes remerciements s'adressent en particulier à Jean Dorey, Frank Debouck, Marie-Annick Galland et Francis Leboeuf, qui nous a quitté en 2013 et auquel nous devons beaucoup. Ils vont aussi vers les nombreux élèves centraliens qui s'impliquent dans les activités en SHS, ce qui rend notre travail pédagogique enrichissant et stimulant. Enfin, je remercie les experts et Sandrine de Montgolfier pour leurs remarques et suggestions sur une première version de ce texte. 